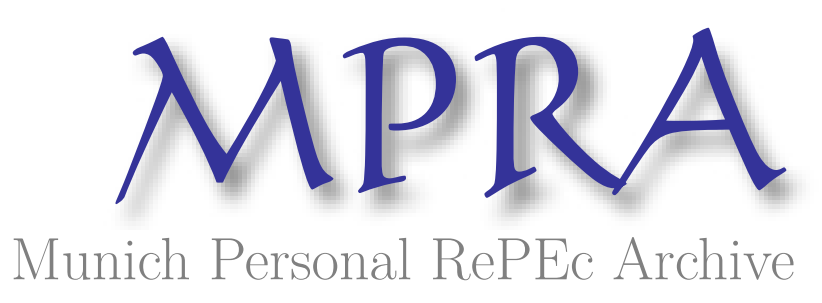

\title{
Unmöglichkeitstheoreme bei asymmetrischer Information
}

Schmitz, Patrick W.

2003

Online at https://mpra.ub.uni-muenchen.de/6934/

MPRA Paper No. 6934, posted 01 Feb 2008 12:48 UTC 


\title{
Unmöglichkeitstheoreme bei asymmetrischer Information*
}

\author{
Patrick W. Schmitz
}

Universität Bonn, 2003

\section{Einleitung}

In diesem Beitrag soll anhand eines einfachen Modells erläutert werden, inwiefern das Vorliegen von privater Information $\mathrm{zu}$ einem Unmöglichkeitstheorem führen kann. Ein Unmöglichkeitstheorem liegt vor, wenn die allokative Entscheidung, die im Fall von symmetrischer Information gefällt werden würde, beim Vorliegen von asymmetrischer Information nicht das Ergebnis freiwilliger Verhandlungen sein kann.

Das hier diskutierte Modell erlaubt vielfältige Interpretationen. Beispielsweise kann man sich vorstellen, daß ein Verkäufer und ein Käufer über den Tausch eines unteilbaren Gutes verhandeln. In diesem Fall würden die beiden Parteien sich im Fall von symmetrischer Information auf den Tausch des Gutes einigen, wenn immer die Zahlungsbereitschaft des Käufers die Kosten des Verkäufers übersteigt. Dies wird als Referenzlösung bezeichnet. Es wird gezeigt, daß es beim Vorliegen von privater Information unmöglich sein kann, die Referenzlösung zu erreichen. Eine andere Interpretation betrifft die Durchführung eines öffentlichen Projektes. Die Zahlungsbereitschaft eines Individuums für die Durchführung kann positiv oder negativ sein. Im Fall von symmetrischer Information würden sich rationale Wirtschaftssubjekte darauf einigen, das Projekt durchzuführen, wenn immer die Summe der Zahlungsbereitschaften positiv ist (dabei können Gewinner Kompensationszahlungen an Verlierer zahlen). Ein Unmöglichkeitsresultat liegt vor, wenn es nicht möglich ist, das Projekt genau dann durchzuführen wenn die Summe der Zahlungsbereitschaften positiv ist.

Ziel des vorliegenden Beitrags ist es, wesentliche Aussagen der relevanten Literatur in einem möglichst einfachen und einheitlichen Modellrahmen zu diskutieren.

\footnotetext{
* Auf dem vorliegenden Diskussionspapier beruht die folgende Publikation:

Schmitz, P.W. (2003), „Unmöglichkeitstheoreme bei asymmetrischer Information“, WiSt Wirtschaftswissenschaftliches Studium, Bd. 32, S. 239-242.
} 


\section{Ein einfaches Modell}

Man betrachte zwei risikoneutrale Vertragsparteien A und B. Es ist eine gemeinsame (kollektive) Entscheidung $x \in\{0,1\}$ zu treffen. Die Entscheidung kann beispielsweise die Produktion bzw. den Tausch eines privaten Gutes oder auch die Bereitstellung eines öffentlichen Gutes betreffen. Gegeben eine Entscheidung $x$ und eine Transferzahlung $t \in \mathfrak{R}$ von Partei B an Partei A lautet der Nutzen von Partei A

$U_{A}=x v+t$

und der Nutzen von Partei B lautet

$U_{B}=x w-t$

Der Parameter $v$, welcher die Zahlungsbereitschaft von Partei A für die Entscheidung $x=1$ gegenüber der Entscheidung $x=0$ bezeichnet, ist private Information von Partei A, während der Parameter $w$ private Information von Partei B ist. Es wird angenommen, daß $v$ einen von zwei Werten annehmen kann. Mit Wahrscheinlichkeit $p$ ist $v=v_{h}$, und mit Wahrscheinlichkeit $1-p$ ist $v=v_{l}$, wobei $v_{l}<v_{h}$. Während die Verteilung von $v$ allgemein bekannt ist, weiß nur Partei A, ob sich die hohe oder die niedrige Zahlungsbereitschaft realisiert hat. Entsprechend ist $w=w_{h}$ mit Wahrscheinlichkeit $q$ und $w=w_{l}$ mit Wahrscheinlichkeit $1-q$, wobei $w_{l}<w_{h}$.

Der soziale Überschuß wird gemessen durch die Summe der Auszahlungen $x(v+w)$. Die ex post effiziente Entscheidung lautet also $x=1$ falls $v+w$ positiv ist und $x=0$ falls $v+w$ negativ ist. Die ex post (also nach der Realisierung von $v$ und $w$ ) effiziente Entscheidung wird auch als Referenzlösung bezeichnet.

Man betrachte zunächst den Fall einseitiger privater Information. Dazu nehme man z.B. an, daß $q=1$ gelte. Dies bedeutet, daß die Zahlungsbereitschaft $w$ von Partei B mit Sicherheit hoch ist, d.h. es sein zunächst immer $w=w_{h}$. Man unterstelle ferner, daß die Entscheidung für den Fall, daß sich die Parteien nicht einigen können, durch $x=0$ gegeben ist. Diese Entscheidung wird als Status Quo Entscheidung bezeichnet. Beispielsweise könnte Partei A 
der Verkäufer einer Einheit eines unteilbaren Gutes sein. Dann bedeutet $x=0$, daß das Gut im Besitz des Verkäufers bleibt, während $x=1$ bedeutet, daß das Gut getauscht wird. Bei dieser Interpretation sind die Kosten des Verkäufers also durch $-v>0$ gegeben. Man beachte, daß der Tausch des Gutes ex post effizient ist, wenn der Nutzen des Käufers die Kosten des Verkäufers übersteigt, d.h. wenn $v+w$ positiv ist.

Es ist offensichtlich leicht möglich, die Referenzlösung zu erreichen, wenn die Entscheidung $x=1$ nie ex post effizient ist $\left(v_{h}+w<0\right)$. In diesem Fall bleibt es sicher beim Status Quo, da zumindest eine Partei jedem anderen Vorschlag widersprechen wird.

Man betrachte nun den interessanteren Fall $-v_{l}>w>-v_{h}$, in dem die ex post effiziente Entscheidung nicht trivial ist, d.h. von der Realisierung von $v$ abhängt: Es soll nur im Fall $\nu=v_{h}$ zur Entscheidung $x=1$ kommen. Es sind sehr viele mögliche Verhandlungsprozesse denkbar. Man nehme beispielsweise an, daß Partei B ein ultimatives Preisangebot machen kann, welches Partei A nur akzeptieren oder ablehnen kann. Partei B wird dann den Preis gleich - $v_{h}$ wählen, so daß sie den gesamten erwarteten Gewinn $p\left(v_{h}+w\right)$ erhält. Wenn hingegen Partei A der Partei B ein ultimatives Angebot machen kann, wird sie im Fall $v=v_{h}$ den Preis $w$ für die Entscheidung $x=1$ verlangen und den gesamten Überschuß für sich beanspruchen. Auf jeden Fall existieren somit Verhandlungsprozesse, die zur Referenzlösung führen.

Schließlich soll der Fall betrachtet werden, in dem die Entscheidung $x=1$ immer ex post effizient ist $\left(v_{l}+w>0\right)$. Falls Partei B der Partei A ein ultimatives Preisangebot machen kann, ist es möglich, daß die Referenzlösung verfehlt wird. Partei B zieht die Preise $-v_{h}$ und $-v_{l}$ in Betracht (also die kleinstmöglichen Zahlungen, welche Partei A akzeptieren wird, falls $v=v_{h}$ bzw. $\left.v=v_{l}\right)$. Der erwartete Gewinn des B beim Preis $-v_{h}$ lautet $p\left(v_{h}+w\right)$, beim Preis $-v_{l}$ lautet der erwartete Gewinn $v_{l}+w$. Es sind also zwei Unterfälle zu unterscheiden. Falls $p\left(v_{h}+w\right)>v_{l}+w$, dann kommt es nur im Fall $v=v_{h}$ zur Entscheidung $x=1$, so daß die Referenzlösung verfehlt wird. Falls $p\left(v_{h}+w\right) \leq v_{l}+w$, dann kommt es immer zu $x=1$, d.h. die Referenzlösung wird erreicht. Man beachte, daß Partei A im ersten Fall keine Rente erhält, während im zweiten Fall ihre erwartete Rente $p\left(v_{h}-v_{l}\right)$ beträgt. Diese Rente erhält Partei A, obwohl Partei B alle Verhandlungsmacht hat. Der Grund für die Rente liegt in der privaten Information des A. Würde der B die Realisierung von $v$ kennen, so könnte er den gesamten Überschuß abschöpfen. Beim Vorliegen von privater Information ist dies jedoch 
nicht der Fall. Die private Information ist also wertvoll für den A, die Rente kann somit als Informationsrente bezeichnet werden.

Falls die Partei A im Fall $v_{l}+w>0$ der Partei B ein ultimatives Angebot machen kann, dann wird sie den Preis gleich $w$ setzen, so daß es immer zum Tausch kommt und die Referenzlösung erreicht wird. Es ist also zusammenfassend in jeder Parameterkonstellation möglich, die Referenzlösung zu erreichen. Im Fall von einseitiger privater Information ist die Referenzlösung immer erreichbar, wenn die informierte Partei der uninformierten Partei ein ultimatives Angebot machen kann, vorausgesetzt die Status Quo Entscheidung ist exogen gegeben. Ob allerdings die Referenzlösung tatsächlich erreicht wird, kann natürlich vom konkreten Verhandlungsprozeß abhängen. Man sagt daher, daß ein Möglichkeitsresultat gilt. Im folgenden sollen demgegenüber zwei Situationen betrachtet werden, in denen die Referenzlösung mit keinem denkbaren Verhandlungsprozeß erreichbar ist, d.h. in denen ein Unmöglichkeitsresultat gilt.

\section{Private Information und endogene Status Quo Entscheidung}

Man betrachte zunächst weiterhin den Fall einseitiger privater Information. Nun soll allerdings unterstellt werden, daß die privat informierte Partei A die Status Quo Entscheidung im Fall der Nichteinigung mit Partei B treffen kann. Die Status Quo Entscheidung ist also im Unterschied zum vorherigen Abschnitt nicht mehr exogen vorgegeben, sondern wird von Partei A endogen bestimmt.

Beispielsweise könnte man sich vorstellen, daß Partei A ein Produzent ist, der durch Einsatz einer neuen Technologie die Emission von Schadstoffen aus seiner Fabrik reduzieren kann. Die Einführung der neue Technologie ist mit Kosten verbunden, führt aber auch zu einer größeren Produktivität. Es ist daher möglich, daß der Produzent die neue Technologie freiwillig einsetzen wird. Es ist jedoch auch möglich, daß die gesteigerte Produktivität nur gering ausfällt, so daß der Produzent die neue Technologie nicht ohne den Erhalt einer Transferzahlung einsetzen wird. Wenn man den positiven externen Effekt, den die neue Technologie aufgrund der besseren Umweltverträglichkeit auf die benachbarte Partei B ausübt, mit berücksichtigt, sei der Einsatz der neuen Technologie in jedem Fall ex post effizient. 
Formal läßt sich die Situation wie folgt beschreiben. Die Entscheidung $x=1$ bedeutet, daß die neue Technologie von Partei A eingesetzt wird. Der Gewinn von Partei A aus dem Einsatz der neuen Technologie (unter Berücksichtigung der Einführungskosten) lautet $v$, wobei $v_{l}<0<v_{h}$ unterstellt wird. Ferner gilt $v_{l}+w>0$, so daß $x=1$ immer ex post effizient ist. Der wichtige Unterschied zum vorherigen Abschnitt besteht darin, daß die Status Quo Entscheidung endogen bestimmt wird: Im Fall $v=v_{l}$ wählt Partei A die Entscheidung $x=0$ und im Fall $v=v_{h}$ die Entscheidung $x=1$, wenn es nicht zu einer Vereinbarung mit Partei B kommt.

Man betrachte nun einen beliebigen Verhandlungsprozeß. Im Gleichgewicht kommt es (gegeben die private Information) $\mathrm{zu}$ einer bestimmten Entscheidung und einer Transferzahlung. Gemäß dem Revelationsprinzip kann man sich nun alternativ vorstellen, daß ein neutraler Vermittler Partei A direkt nach ihrer privaten Information fragt. In Abhängigkeit von der Antwort wird eine Entscheidung und eine Transferzahlung vorgeschrieben. Dies wird auch als direkter Mechanismus bezeichnet. Partei A wird wahrheitsgemäß antworten, wenn der Vermittler genau die Entscheidung und Transferzahlung vorschreibt, die sich im Gleichgewicht (gegeben die private Information) bei dem ursprünglichen Verhandlungsspiel herausgestellt hätte. Wenn es also keinen ex post effizienten direkten Mechanismus gibt, der Partei A dazu bringt, die Wahrheit zu sagen, so kann überhaupt kein Verhandlungsprozeß existieren, der die Referenzlösung implementiert.

Es stellt sich nun die Frage, ob die ex post effiziente Entscheidung ( $x \equiv l)$ mit Hilfe eines Transferschemas $\left(t_{l}, t_{h}\right)$ erreichbar ist, welches Zahlungen in Abhängigkeit von der Antwort der Partei A vorschreibt. Partei A sagt nur dann die Wahrheit, wenn die Anreizverträglichkeitsbedingungen

$v_{l}+t_{l} \geq v_{l}+t_{h}$ und $v_{h}+t_{h} \geq v_{h}+t_{l}$

erfüllt sind. Die erste Bedingung besagt, daß es sich für Partei A nicht lohnen darf, im Fall $v=v_{l}$ zu behaupten, daß $v=v_{h}$ gilt. Die zweite Bedingung gilt analog für den Fall $v=v_{h}$. Dies bedeutet aber, daß die Transferzahlung konstant sein muß, $t_{l}=t_{h}=t$. 
Partei A ist im Fall eines niedrigen Gewinns nur dann bereit, an dem vorgeschlagenen Mechanismus teilzunehmen, wenn die Bedingung der individuellen Rationalität

$v_{l}+t \geq 0$

erfüllt ist. Partei B kennt die Realisierung von $v$ nicht und geht somit davon aus, daß Partei A im Fall der Nichteinigung mit Wahrscheinlichkeit $p$ die Entscheidung $x=1$ trifft. Die Teilnahmebedingung von B lautet also

$w-t \geq p w$.

Aus diesen Bedingungen folgt, daß die gesuchte Transferzahlung nicht existieren kann, falls

$-v_{l}>(1-p) w$

gilt, was in der unterstellten Parameterkonstellation nicht ausgeschlossen ist. In diesem Fall kann also die Referenzlösung mit keinem denkbaren Verhandlungsprozeß erreicht werden. Dieses Resultat läßt sich verallgemeinern. Der interessierte Leser sei hierzu auf den Artikel von Klibanoff/Morduch (1995) verwiesen. Man beachte, daß das in diesem Abschnitt hergeleitete Unmöglichkeitstheorem gilt, obwohl es allgemein bekannt ist, daß die ex post effiziente Entscheidung immer $x=1$ lautet. Allerdings hängt das Resultat entscheidend davon ab, daß die Status Quo Entscheidung durch die Entscheidung gegeben ist, welche für Partei A optimal ist, wenn es zu keiner Vereinbarung mit Partei B kommt. Könnte Partei A glaubhaft drohen, nur dann $x=1$ zu wählen, wenn sie von Partei B eine Zahlung in Höhe von $w$ bekommt, so wäre die Referenzlösung erreichbar. Im folgenden Abschnitt soll ein Unmöglichkeitsresultat diskutiert werden, welches nicht von einer endogenen Status Quo Entscheidung abhängt.

\section{Zweiseitige private Information}

Man betrachte nun eine Situation mit zweiseitiger privater Information, d.h. sowohl $p$ als auch $q$ liegen strikt zwischen 0 und 1. Die Status Quo Entscheidung sei exogen durch $x=0$ gegeben. Beispielsweise könnte $x=1$ bedeuten, daß Partei A ein privates Gut an Partei B 
abgibt (dann ist $v$ immer negativ und $w$ immer positiv), oder daß ein öffentliches Projekt durchgeführt wird, welches von den Parteien positiv oder negativ bewertet werden kann.

Zunächst läßt sich leicht sehen, daß die Referenzlösung immer erreichbar ist, wenn die ex post effiziente Lösung nicht von der Realisierung der privaten Information abhängt. Wenn beispielsweise $-v_{l}>-v_{h}>w_{h}>w_{l}$ gilt, dann ist $v+w$ immer negativ, d.h. die ex post effiziente Entscheidung entspricht dem Status Quo und ist offensichtlich erreichbar. Wenn $w_{h}$ $>w_{l}>-v_{l}>-v_{h}$ gilt, dann ist $v+w$ immer positiv und die ex post effiziente Entscheidung lautet $x=1$. Die Referenzlösung läßt sich durch einen Mechanismus erreichen, der vorschreibt, daß $x=1$ genau dann implementiert wird, wenn Partei B eine Zahlung an Partei A leistet, welche zwischen $-v_{l}$ und $w_{l}$ liegt (man beachte, daß diese Zahlung negativ sein kann, in diesem Fall zahlt also Partei A an Partei B einen positiven Betrag). Offenbar ziehen beide Parteien diesen Mechanismus, der immer zu $x=1$ führt, dem Status Quo vor.

Es gibt jedoch auch Parameterkonstellationen, in denen es von der Realisierung der privaten Information abhängt, welche Entscheidung ex post effizient ist. Falls $w_{h}>-v_{l}>-v_{h}>w_{l}$ gilt, läßt sich die Referenzlösung erreichen, falls analog zum soeben beschriebenen Mechanismus die Zahlung von $\mathrm{B}$ an $\mathrm{A}$ zur Herbeiführung der Entscheidung $x=1 \quad$ zwischen $-v_{l}$ und $w_{h}$ liegt (der Mechanismus führt immer dann zu $x=1$, wenn $w=w_{h}$ gilt, was ex post effizient ist). Im Fall $-v_{l}>w_{h}>w_{l}>-v_{h}$ muß die Zahlung entsprechend zwischen $-v_{h}$ und $w_{l}$ liegen, und im Fall $-v_{l}>w_{h}>-v_{h}>w_{l}$ zwischen $-v_{h}$ und $w_{h}$.

Interessant ist nun die verbleibende Konstellation $w_{h}>-v_{l}>w_{l}>-v_{h}$. In diesem Fall ist es möglich, daß die Referenzlösung nicht erreichbar ist. Dies soll anhand eines Beispiels illustriert werden: Sei $p=q=1 / 2$. Das Unmöglichkeitsresultat wird wiederum durch Rückgriff auf das Revelationsprinzip bewiesen. Wenn es ein Verhandlungsspiel gibt, welches die ex post effiziente Lösung implementiert, so gibt es auch einen ex post effizienten direkten Mechanismus, bei dem A und B ihre Zahlungsbereitschaften wahrheitsgemäß enthüllen (dieser Mechanismus schreibt im Gleichgewicht die Entscheidung und die Zahlung vor, die sich im Gleichgewicht des ursprünglichen Verhandlungsspiels ergeben hätten). Bezeichne $t_{i j}$ die Zahlung von Partei B an Partei A, falls Partei A behauptet, daß $v=v_{i}$ ist, und Partei B behauptet, daß $w=w_{j}$ ist, wobei $i, j \in\{l, h\}$. Man beachte, daß in der betrachteten Konstellation die Entscheidung $x=1$ immer ex post effizient ist, außer im Fall $v=v_{l}, \quad w=w_{l}$. 
Im Fall $v=v_{h}$ sagt Partei A nur dann die Wahrheit, falls die folgende Anreizverträglichkeitsbedingung gilt:

$1 / 2\left(v_{h}+t_{h h}\right)+1 / 2\left(v_{h}+t_{h l}\right) \geq 1 / 2\left(v_{h}+t_{l h}\right)+1 / 2 t_{l l}$

Diese Bedingung besagt, daß es sich für den A nicht lohnt $v=v_{l}$ zu behaupten, falls B die Wahrheit sagt. Analog verlangt die Anreizverträglichkeitsbedingung von Partei B für den Fall $w=w_{h}:$

$1 / 2\left(w_{h}-t_{h h}\right)+1 / 2\left(w_{h}-t_{l h}\right) \geq 1 / 2\left(w_{h}-t_{h l}\right)-1 / 2 t_{l l}$

Aus diesen beiden Bedingungen folgt durch Addition und Umformung:

$1 / 2\left(v_{h}+w_{h}\right) \geq t_{l h}-t_{h l}$

Ferner ist zu berücksichtigen, daß die Teilnahme für die Parteien individuell rational sein muß. Im Fall $v=v_{l}$ verlangt die Teilnahmebedingung für Partei A:

$1 / 2\left(v_{l}+t_{l h}\right)+1 / 2 t_{l l} \geq 0$

Die Teilnahmebedingung für Partei B lautet für den Fall $w=w_{l}$ entsprechend:

$1 / 2\left(w_{l}-t_{h l}\right)-1 / 2 t_{l l} \geq 0$

Addition dieser beiden Teilnahmebedingungen und Umformung ergibt:

$t_{l h}-t_{h l} \geq-v_{l}-w_{l}$

Zusammenfassen bedeutet dies aber, daß die gesuchten Transferzahlungen nicht existieren können, falls

$-v_{l}-w_{l}>1 / 2\left(v_{h}+w_{h}\right)$ 
gilt, was in der betrachteten Konstellation $w_{h}>-v_{l}>w_{l}>-v_{h}$ durchaus nicht ausgeschlossen ist. In diesem Fall ist es somit unmöglich, die Referenzlösung zu erreichen. Auch dieses Resultat läßt sich wiederum verallgemeinern. Das bekannteste Unmöglichkeitstheorem für den Fall der bilateralen privaten Information stammt von Myerson/Satterthwaite (1983), deren wichtiger Artikel Ausgangspunkt der umfangreichen Literatur war, in die der vorliegende Beitrag einige Einblicke vermittelt hat.

\section{Fazit}

Im Fall von einseitiger privater Information und einer exogen vorgegebenen Status Quo Entscheidung läßt sich die Referenzlösung erreichen, wenn die informierte Partei der uninformierten Partei ein ultimatives Angebot machen kann. Es kann jedoch unmöglich sein, die Referenzlösung zu erreichen, falls im Fall einseitiger privater Information die Status Quo Entscheidung endogen von der informierten Partei getroffen wird, oder falls im Fall einer exogenen Status Quo Entscheidung zweiseitige private Information vorliegt und die ex post effiziente Entscheidung in nicht trivialer Weise von der Realisierung der privaten Information abhängt.

\section{Literatur}

Klibanoff, P., J. Morduch, Decentralization, Externalities, and Efficiency, in: Review of Economic Studies, Vol. 62 (1995), S. 233-247.

Myerson, R.B., M.A. Satterthwaite, Efficient Mechanisms for Bilateral Trading, in: Journal of Economic Theory, Vol. 29 (1983), S. 265-281. 